\title{
PKS 1814-637: a powerful radio-loud AGN in a disk galaxy
}

\author{
R. Morganti ${ }^{1,2}$, J. Holt ${ }^{3}$, C. Tadhunter ${ }^{4}$, C. Ramos Almeida ${ }^{4}$, D. Dicken ${ }^{5}$, K. Inskip ${ }^{6}$, T. Oosterloo ${ }^{1,2}$, and T. Tzioumis ${ }^{7}$
}

\author{
1 Netherlands Foundation for Research in Astronomy, Postbus 2, 7990 AA, Dwingeloo, The Netherlands \\ e-mail: morganti@astron.nl \\ 2 Kapteyn Astronomical Institute, University of Groningen, PO Box 800, 9700 AV Groningen, The Netherlands \\ 3 Leiden Observatory, Leiden University, PO Box 9513, 2300 RA Leiden, The Netherlands \\ ${ }^{4}$ Dep. Physics and Astronomy, University of Sheffield, Sheffield, S7 3RH, UK \\ 5 Department of Physics and Astronomy, Rochester Institute of Technology, 84 Lomb Memorial Drive, Rochester NY 14623, USA \\ 6 Max-Planck-Institut für Astronomie, Königstuhl 17, 69117 Heidelberg, Germany \\ 7 CSIRO Australia Telescope National Facility, PO Box 76, 1710 Epping NSW, Australia
}

Received 12 July 2011 / Accepted 31 August 2011

\begin{abstract}
We present a detailed study of PKS 1814-637, a rare case of powerful radio source $\left(P_{5 \mathrm{GHz}}=4.1 \times 10^{25} \mathrm{~W} \mathrm{~Hz}^{-1}\right)$ hosted by a disk galaxy. Optical images have been used to model the host galaxy morphology confirming it to be dominated by a strong (and warped) disk component that is observed close to edge-on to the line of sight. This is the first radio galaxy found to reside in a disk dominated galaxy with radio luminosity equivalent to powerful FRII objects. At radio wavelengths, PKS 1814-637 is about 480 pc in diameter and it is classified as a compact steep spectrum (CSS) source; such sources are usually considered to be radio sources observed in the early stages of their evolution. However, the optical and mid-IR spectroscopic properties of PKS1814-637 show more in common with Seyfert galaxies than they do with radio galaxies, with the detection of $\mathrm{H}_{2}$, and $\mathrm{PAH}$ emission features, along with $\mathrm{HI}$ and silicate absorption features, providing evidence for a rich ISM which is likely to be related to the disk morphology of the host galaxy. We argue that the interaction between the radio plasma and the rich ISM in this and similar objects may have boosted their radio emission, allowing them to more easily enter flux limited samples of radio sources. In this case, PKS 1814-637 represents a type of "imposter": an intrinsically low power object that is selected in a radio flux limited sample because of the unusually efficient conversion of jet power into radio emission. This would make PKS 1814-637 an extreme example of the effects of jet-cloud interactions in galaxies containing a rich ISM, and perhaps a missing link between radio galaxies and radio-loud Seyfert galaxies. However, it is unlikely that jet-cloud interactions alone can account for the unusually powerful radio emission compared to Seyfert galaxies, and it is probable that the jet in PKS 1814-637 is also intrinsically more powerful than in typical Seyfert galaxies, perhaps due to a higher bulge and black hole mass. The estimated BH mass is indeed higher than the majority of Seyfert galaxies in the local Universe. We speculate that sources similar to PKS1814-637 are likely to be more common at high redshifts, because of the enhanced probability at earlier epochs of triggering radio sources in moderately massive bulges that are also gas-rich.
\end{abstract}

Key words. galaxies: active - galaxies: individual: PKS 1814-637 - ISM: jets and outflows - radio lines: galaxies

\section{Introduction}

The origin of the dichotomy between radio-quiet and radio-loud objects is still a matter of debate: is it a question of nature or of nurture? Both intrinsic differences in the central engine and extrinsic differences in the surrounding medium have been considered. It is intriguing that optical AGN (Seyfert-like type) are mainly found in late-type, disk galaxies (both in the local as in the far away Universe, see e.g. Schawinski et al. 2011), while this is not the case for radio-loud AGN. These are mainly hosted by early-type galaxies (Best et al. 2005, and references therein).

The fact that Seyfert galaxies do have, on average, radio core powers lower than those of radio galaxies suggests that they are weaker radio sources from the outset (see e.g. Bicknell et al. 1998) and points, therefore, to a connection with intrinsic differences e.g. BH mass and/or spin. However, the characteristics of the inter-stellar medium (ISM) in which a radio source is born appear also to play a role in its further evolution. This suggests, for example, that entrainment by the radio jet may have some influence on the level of kiloparsec-scale radio emission (Bicknell et al. 1998) and/or that in radio sources hosted by spiral galaxies, even initially relativistic jets can be rapidly decelerated by collisions in a dense surrounding broad line region or ISM (see e.g. Taylor et al. 1989). Connected to this, the possibility that the radio emission could actually be temporarily enhanced by such interactions, see e.g. (Gopal-Krishna \& Wiita 1991), further complicates our classification of radio sources.

Although it is extremely rare for nearby radio galaxies to be hosted by genuine disk galaxies, a few examples do exist that have been studied in detail (see Table 1 for a summary). For example, the spiral galaxy 0313-192 in the Abell cluster A428 hosts a large double-lobed Fanaroff-Riley I (FR I) radio source (Ledlow et al. 1998, 2001; Keel et al. 2006), while NGC 612 is an S0 galaxy with a powerful FR-I/FR-II hybrid radio source and a large-scale star-forming H I disk (Véron-Cetty \& Véron 2001; Emonts et al. 2008). B2 $0722+30$ is another FR I radio galaxy whose jets are mis-aligned to the galaxy disk, but appear to be aligned with an $\mathrm{HI}$ bridge towards a nearby, interacting pair of galaxies (Emonts et al. 2009). Other possible examples include 3C 293 (van Breugel et al. 1984) and 3C 305 (Heckman et al. 1982), whose host galaxy morphologies are highly disturbed due to recent galaxy interactions, but show disk-like characteristics. 
Table 1. The properties of powerful radio radio sources hosted by disk galaxies.

\begin{tabular}{lccc}
\hline \hline Object & Redshift $(z)$ & $\begin{array}{c}P_{5 \mathrm{GHz}} \\
\mathrm{W} \mathrm{Hz}^{-1}\end{array}$ & Reference \\
\hline NGC 612 & 0.0298 & $8 \times 10^{24}$ & 1 \\
0313-192 & 0.0671 & $9 \times 10^{23}$ & $2,3,4$ \\
B2 0722+30 & 0.0188 & $6 \times 10^{22}$ & 4,5 \\
3C293 & 0.0450 & $9 \times 10^{24}$ & 6 \\
3C305 & 0.0416 & $4 \times 10^{24}$ & 7 \\
Speca & 0.1378 & $3 \times 10^{24 *}$ & 8 \\
PKS 1814-637 & 0.0641 & $4 \times 10^{25}$ & 9 \\
\hline
\end{tabular}

Notes. ${ }^{*}$ Total flux derived from NVSS at $1.4 \mathrm{GHz}$ and extrapolated to $5 \mathrm{GHz}$ assuming $\alpha=0.7$.

References. 1. Emonts et al. (2008); 2. Ledlow et al. (2001); 3. Keel et al. (2006); 4. NASA Extragalactic Database; 5. Emonts et al. (2009); 6. van Breugel et al. (1984); 7. Heckman et al. (1982); 8. Hota et al. (2011); 9. this paper.

An other very recent example is the intermediate redshift disklike objects that shows spectacular radio lobe structures on scales of several $100 \mathrm{kpc}$ suggestive of relicts Hota et al. (2011).

These rare cases of disk-dominated radio galaxies provide an excellent opportunity for studying the host galaxy properties and environmental effects that could be important for the triggering and/or evolution of their radio sources. In addition, a detailed knowledge of these systems provides valuable information for a comparison with studies at high redshift. Some of these studies are now in progress, exploring whether disk-like host galaxies may be much more common at high than at low redshift (Norris et al. 2008). However, much more will be done in the near future, due to planned large radio and optical surveys.

In this paper we present a detailed study of a newly found, extreme example of a radio source hosted by a disk galaxy (see Fig. 1). Unlike all objects mentioned above, the radio power of PKS 1814-637 $\left(P_{5 \mathrm{GHz}}=4.1 \times 10^{25} \mathrm{~W} \mathrm{~Hz}^{-1}\right.$, Tadhunter et al. 1993; Morganti et al. 1993, 2001) falls well above the radio power boundary between FRI and FRII radio sources $\left(P_{5 \mathrm{GHz}} \sim\right.$ $10^{25} \mathrm{~W} \mathrm{~Hz}^{-1}$; Fanaroff \& Riley 1974). For comparison, its radio power is two orders of magnitude higher than the most powerful radio Seyfert galaxy (NGC 1068, $P_{5} \mathrm{GHz} \sim 10^{23} \mathrm{~W} \mathrm{~Hz}^{-1}$ ), and more than a factor of four higher than the next most powerful radio source hosted by a disk galaxy (see Table 1). Interestingly, radio-loud narrow line Seyfert 1 (NLS1) galaxies can reach radio power comparable to PKS 1814-637 (see e.g. Foschini 2011, and references therein) but because their radio emission is likely dominated by a beamed jet emission, their intrinsic jet (and extended radio lobe powers) might be orders of magnitude lower, making them more similar to Seyferts or low power FRIs. Because of all this, PKS 1814-637 stands out as a particularly interesting object.

From the radio perspective, PKS 1814-637 is a compact steep spectrum (CSS) radio source (Tzioumis et al. 2002), see also Fig. 1, right), of about 480 pc linear size ${ }^{1}$. The consensus is that the majority of such sources are young radio sources. However, this class can also include cases that are unable to become large due to confinement of the radio source by the dense ISM (see e.g. Reynolds \& Begelman 1997; KunertBajraszewska et al. 2004; Orienti et al. 2010, and references

\footnotetext{
1 Throughout this paper we use a Hubble constant $H_{0}=$ $70 \mathrm{~km} \mathrm{~s}^{-1} \mathrm{Mpc}^{-1}$ and $\Omega_{\Lambda}=0.7$ and $\Omega_{\mathrm{M}}=0.3$. At the distance of PKS 1814-637 this results in 100 mas $=120$ pc, Wright (2006).
}

therein). Finally, HI in absorption with high optical depth has been detected in PKS 1814-637 (Véron-Cetty et al. 2000; Morganti et al. 2001) and has motivated the VLBI follow up that is presented in this paper.

Here we will use new optical, IR and HI observations of PKS1814-637 to investigate the morphology of the host galaxy, study its ISM, and attempt to understand why such a strong radio source is located in this unusual host.

The paper is organised in the following way. In Sect. 2 we characterise the optical morphology by analysing deep Gemini optical images recently presented in Ramos Almeida et al. (2011) as part of a larger deep optical imaging survey of the $2 \mathrm{Jy}$ sample. In Sect. 3, we derive an accurate systemic velocity of the galaxy by analysing previously unpublished near-IR spectroscopic data and by reanalysing our optical spectroscopy results (Holt et al. 2008). We also include an investigation of the emission line kinematics and discuss the optical spectral classification of the AGN. In Sect. 5 we discuss our recent mid-IR Spitzer data (see also Dicken et al. 2011) and in Sect. 6 we discuss new radio VLBI observations obtained with the Australian Long Baseline Array (LBA). We bring all the results together in Sect. 6 and we further discuss the implication for high- $z$ observations in Sect. 7.

\section{Host galaxy morphology}

Although the morphology of the host galaxy of PKS 1814-637 already appears to be exceptional when compared to other radio galaxies (cf. deep imaging of the 2 Jy sample presented in Ramos Almeida et al. 2011), we now quantify this via modelling the optical morphology.

Deep optical broad $\left(r^{\prime}\right)$ band images of PKS 1814-637 were obtained using the Gemini Multi-Object Spectrograph South (GMOS-S) on the 8.1-m Gemini South telescope at Cerro Pachón, Chile (see Fig. 1). For a full discussion of the observations and data reduction procedure we refer readers to Ramos Almeida et al. (2011).

We have modelled the optical image using GaLFIT (Peng et al. 2002, 2010; version 3.0$)^{2}$. This has been a challenging process because of the dust lane, as well as the presence of the bright foreground star 1.53 arcsec in projection from the nucleus of the radio galaxy. Shorter exposure time images were used in the modelling in order to avoid saturation effects. We derived a PSF profile by extracting 2D images of stars in the GMOS-S image, normalizing to unit flux and taking an average profile weighted by the signal-to-noise ratio of the component extracted stellar profiles. The host galaxy was modelled over a $84 \times 84 \mathrm{kpc}^{2}$ area using a Sérsic profile (Sérsic 1963), and two Gaussian components for fitting the foreground star and the unresolved nuclear point source emission from the AGN. All model parameters, including the host galaxy, star and AGN centroids were allowed to vary freely. We also left the residual background level as an additional free parameter. In order to obtain a reasonable fit, it was necessary to mask the dust lane. Since the galaxy is in a crowded field, we also iteratively modelled all neighbouring stars and galaxies that interfere with the host galaxy model fit.

\footnotetext{
${ }^{2}$ GALFIT is a well-documented two-dimensional fitting algorithm which allows the user to simultaneously fit a galaxy image with an arbitrary number of different model components, in order to extract structural parameters of the galaxy. The model galaxy is convolved with a point spread function (PSF) and, using the downhill-gradient LevenbergMarquardt algorithm, is matched to the observational data via the minimization of the $\chi^{2}$ statistic.
} 


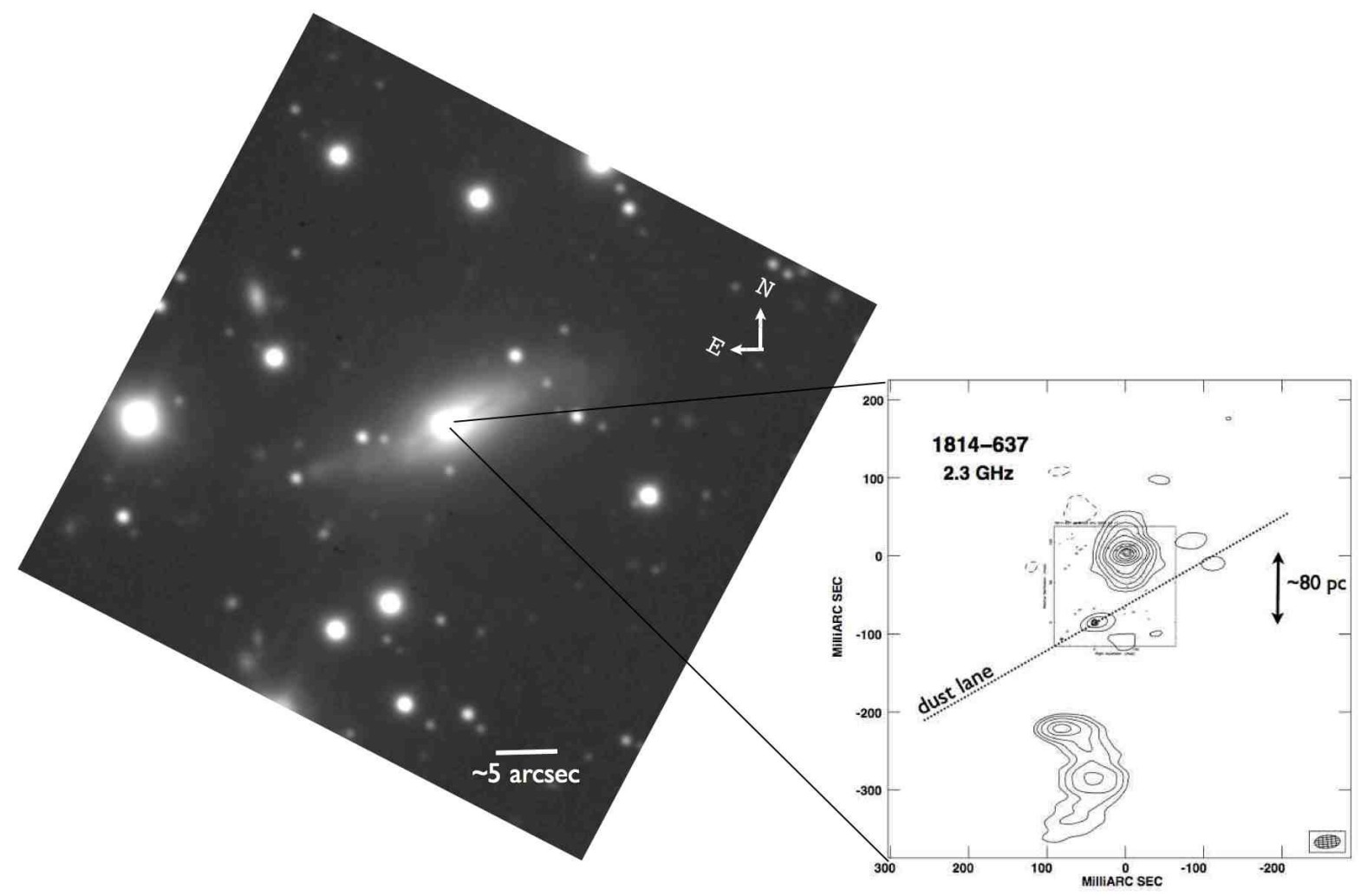

Fig. 1. Left: optical $r^{\prime}$-band GMOS-S image of PKS 1814-637 obtained from the Gemini South. The bright peak coincides with a foreground star. Despite the presence of the star, the extended disky component and dust-lane structure in the galaxy are clearly visible. Right: composite radio map showing the $2.3 \mathrm{GHz}$ VLBI image (Tzioumis et al. 2002) with the $8.4 \mathrm{GHz}$ (i.e. high resolution) image from Ojha et al. 2004) superimposed. This overlay illustrates the presence of a compact component ( $\mathrm{SE}$ of the brighter lobe) that becomes prominent in the high frequency observations: we identify this component with the radio core. The image at $2.3 \mathrm{GHz}$ was obtained from the SHEVE array, the peak a level is $1.7 \mathrm{Jy}$ and contours are shown at $-1.5,1.5,3,6,12,18,35,50,65,80 \%$ of the peak (from Tzioumis et al. 2002).

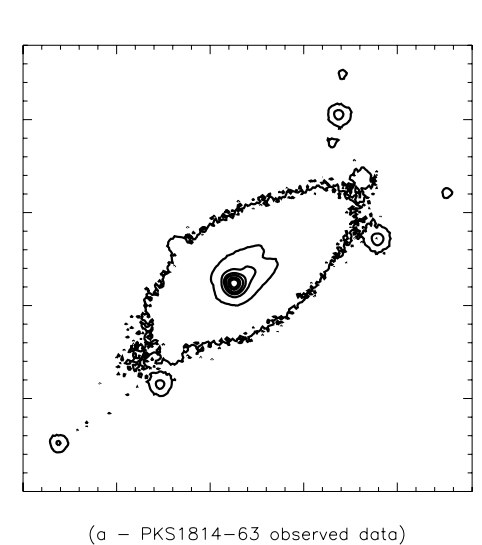

(a)

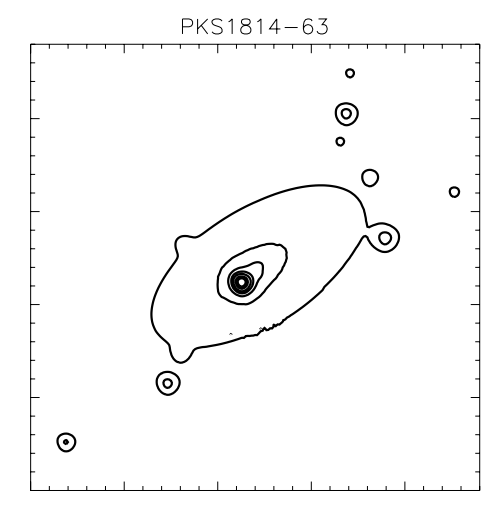

(b)

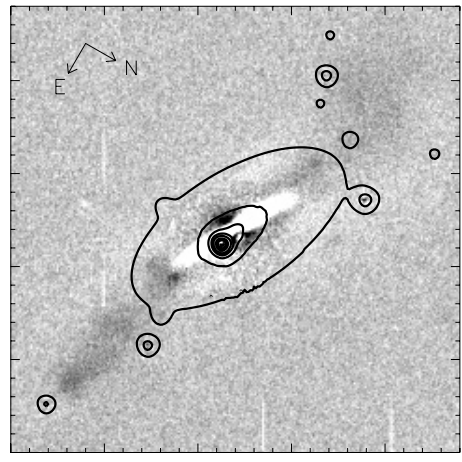

(c)

Fig. 2. GALFIT modelling results. a) Gemini optical $r^{\prime}$-band GMOS-S image contours of PKS1814-637, b) best fitting GALFIT model and c) residuals. See Section 2 for details. Note that the orientation of these images differs from that of the image displayed in Fig. 1.

Once a good model for these adjacent objects had been obtained, their parameters were held fixed, effectively removing them from consideration. The final reduced- $\chi^{2}$ value was determined by repeat modelling all other objects in the field of view, resulting in an ideal value of 1.053 .

The best fit to the optical image of PKS 1814-637 is a Sérsic profile with index $n=2$ which is shown in Fig. 2. Models using more elliptical profiles (e.g. Sérsic $n=4$ ) fail to fit the optical image of PKS 1814-637. The estimated effective radius of the single $n=2$ model is $R_{\text {eff }}=6.4 \mathrm{kpc}$, and the position angle of its major axis PA $=-57^{\circ}$, ellipticity $b / a=0.48$, and magnitude $r^{\prime}=15.75 \mathrm{mag}$. The AGN magnitude was found to be $r^{\prime}=20.98 \mathrm{mag}$ and that of the foreground star $r^{\prime}=15.47$ mag. Other models were tried, such as excluding the very faint 


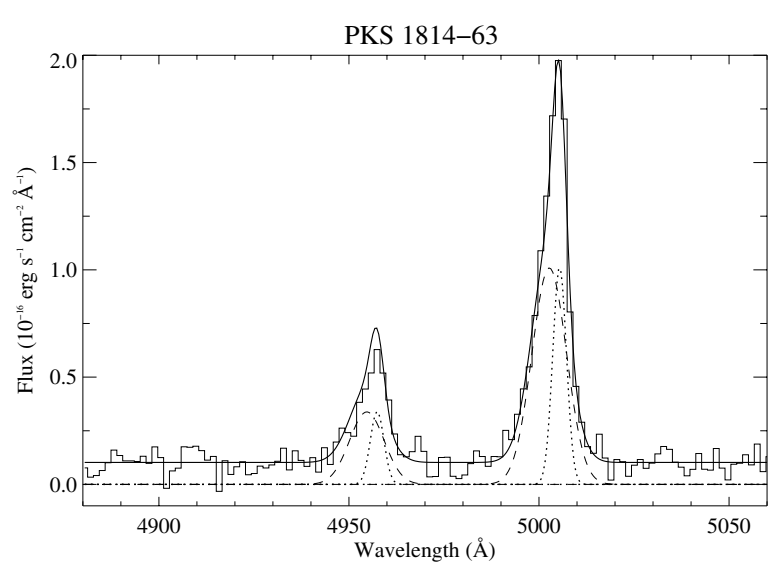

Fig. 3. Model to the $[\mathrm{O} I I I] \lambda \lambda 4959,5007$ emission-line doublet in the nuclear aperture. The solid lines represent the star-subtracted spectrum and the overall model fit to the doublet. The components are: narrow (dotted) and broad (dashed). The two components are overplotted on the radial velocity profile in Fig. 4.

AGN component and a two-component, Sérsic fit. None of these fits gave a better fit to the data and so the simplest model providing a good fit is preferred. Figure 2 shows the contour plots of only the central $50 \times 50 \mathrm{kpc}^{2}$ of the modelled region, together with contours of the best-fitting model overlaid on a grey-scale of the model-subtracted residual images in the same region. The residuals of the fit clearly show the central dust lane, aligned with a disk of $\sim 45 \mathrm{kpc}$ in length, which is heavily warped at the extremes; the fact that the dust lane intercepts the bulge of the galaxy close to the position of the nucleus implies that the inner disk of the galaxy is observed close to edge-on $\left(i>80^{\circ}\right)$.

To summarise, GALFIT modelling is consistent with PKS 1814-637 having a strong disk morphology, confirming the results based on visual examination of the images. In addition, the heavily warped outer parts of the disk provide evidence that the galaxy has undergone a recent galaxy interaction, perhaps related to the triggering of the activity.

\section{Re-deriving the systemic velocity}

In order to understand the role of the various gaseous components in PKS 1814-63, it is essential to determine an accurate systemic velocity. Like the morphological study, spectroscopic investigation of PKS1814-637 is complicated by the presence of the bright foreground star close to the nucleus of the galaxy. As shown in Holt et al. (2008), the nuclear emission line profiles can be modelled using two Gaussian components (see also Fig. 3). The narrowest component is spectroscopically unresolved, and the broader component has a $F W H M$ of $569 \pm 35 \mathrm{~km} \mathrm{~s}^{-1}$.

As for many other sources in their compact radio sources sample, Holt et al. (2008) assumed that the optical narrow component in PKS1814-637 represents the systemic velocity. However, this result has always been considered uncertain: only half of the optical radial velocity profile (or "rotation curve") is observed due to the presence of the bright foreground star, which completely dominates the flux on one side of the galaxy (see Fig. 1 of Holt et al. 2008; and Fig. B9 of Holt et al. 2009). Here we re-analyse the optical spectroscopic data with the help of other available data.

Figure 4 shows the redshifts derived from the two optical components from Holt et al. (2008) and from the deep and shallow H I components from Morganti et al. (2001), see also Sect. 6

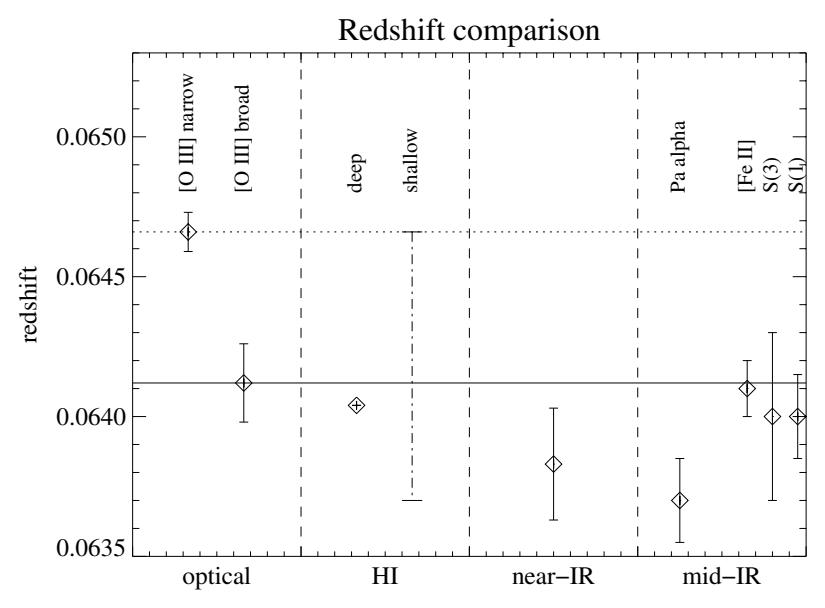

Fig. 4. Comparison of the redshifts derived from the various observations. The horizontal lines represent the original (dotted; from Holt et al. 2008) and new (solid; this paper) interpretations of the systemic velocity. The vertical dot-dashed line labelled "shallow" marks the range of velocities over which the shallow H I absorption is observed (Morganti et al. 2001). The data are also summarised in Table 1.

for more details. Also plotted are the redshifts derived from new Spitzer data (see Sect. 5) and near- IR data from NTT/SOFI (M. Bellamy, priv. comm.). Combining the new data and the original optical data, it is clear from Fig. 4 that a more likely interpretation of the kinematics is that the detected optical broad component is consistent with the systemic velocity, as this is also consistent with all other measured redshifts. While the optical broad component has a relatively large FWHM, this can be explained in terms of unresolved rotation in the inner disk. In this interpretation, the optical narrow component is associated with the (quiescent) disk of the galaxy rotating away from the observer on the west side of the galaxy; the rotation of the large-scale disk is not clearly detected on the east side of the nucleus due to the bright foreground star, but it is detected in the nuclear aperture along with the broad component (see Fig. 3). The failure to detect a blueshifted narrow component in the nuclear aperture (corresponding to the part of the extended disk rotating towards us) is likely to be due to a combination of uneven gas emission and dust obscuration.

Hence, in our new kinematic interpretation, no nuclear outflow is observed in this source, making it unusual amongst compact radio sources (cf. Holt et al. 2008). The new, heliocentric corrected systemic redshift of PKS 1814-637 is $0.06412 \pm$ 0.00014 . The kinematic offsets of the various components have been calculated with respect to this new systemic redshift and are presented in Table 2 .

\section{Optical spectral classification}

Recently there has been much speculation that the optical spectral classification of a radio galaxy is strongly correlated to the rate or mode of accretion of material onto its central supermassive black hole. Radio galaxies with strong optical emission lines (also labelled as "high excitation galaxies", HEGs), including narrow line radio galaxies (NLRG), broad line radio galaxies (BLRG) and quasars, are thought to be energised by the accretion of cool/warm material via a thin accretion disk. On the hand, weak line radio galaxies (sometimes labelled as "low excitation galaxies", LEGs) may be powered by the Bondi accretion of the hot phase of the ISM. Details about this classification can be 


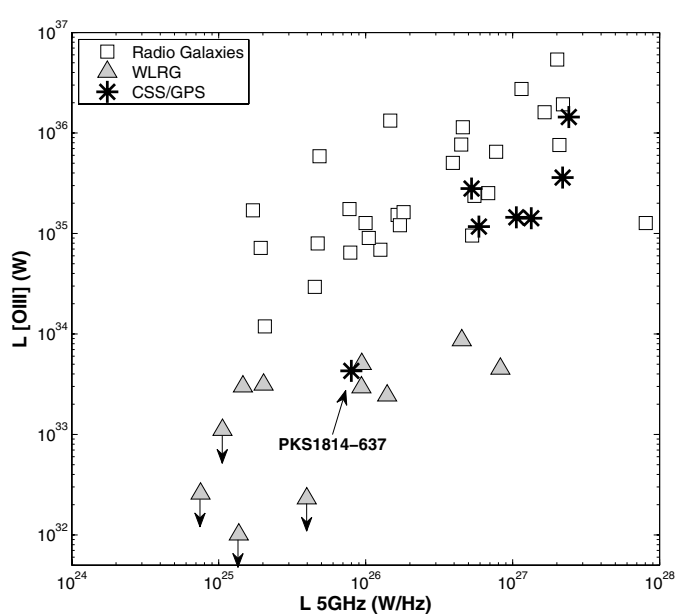

Fig. 5. Plot of [O III] luminosity versus $5 \mathrm{GHz}$ radio power. The points represent radio sources from the 2 Jy sample (Dicken et al. 2011), with the CSS/GPS objects marked as stars.

found e.g. in Hardcastle et al. (2006); Buttiglione et al. (2010). In this context, it is interesting to consider the optical spectral classification of PKS 1814-637.

In terms of its emission line luminosity, PKS 1814-637 falls (by an order of magnitude) well below the correlation between emission line luminosity and radio power (see Fig. 5). In this sense it is similar to the WLRG, that are defined to have small [OIII] $] 5007$ emission line equivalent widths $\left(E W_{\text {[OIII] }}<10 \AA\right.$, see Tadhunter et al. 1998). However, in contrast to the other WLRG in the 2 Jy sample, our previous spectroscopic investigation of PKS 1814-637 (see Holt et al. 2008) suggested a higher equivalent width and classification as a NLRG.

Unfortunately, our previous estimate of the equivalent width derived from the long-slit spectrum was potentially hampered by the (uncertain) subtraction of the continuum associated with the bright star near the nucleus. Therefore we have re-estimated it using better data. Our Gemini images, which have good seeing, allow more reliable subtraction of the star and determination of the galaxy continuum flux in the aperture used for the spectroscopic observations. They confirm that the nuclear continuum level is similar to that in the spectrum presented in Holt et al. (2008). Combining this information with the most reliable estimate of the [O III] emission line flux (Tadhunter et al. 1993), we find that PKS 1814-637 has an [O III] equivalent width in the range 50-100 $\AA$, whereas we define WLRG to have $E W_{\text {[OIII] }}<$ $10 \AA$. Therefore this object is truly ambiguous: it appears like a NLRG in terms of [O III] $E W$, but more like a WLRG in terms of the [OIII] emission line luminosity; PKS 1814-637 is classified as a NLRG, despite its low $L_{[\mathrm{OIII}]}$, because it has an unusually low (stellar) continuum flux. We can naturally link this with the unusual morphology of the host galaxy: a disk galaxy with a relatively low central surface brightness, rather than an elliptical with a high central surface brightness.

It is interesting that the emission line luminosity of PKS 1814-637 (and indeed other WLRG) falls within the range measured for Seyfert galaxies in the local Universe. This leads to the intriguing conclusion that if it were not for the radio data, PKS 1814-637 would have been classified as a typical Seyfert galaxy in terms of its optical and mid-IR spectra (see below), and optical morphology.

\section{The ISM of PKS 1814: Spitzer mid-IR data}

Figure 6 shows the Spitzer IRS spectrum obtained as part of our multi-wavelength investigation of the 2 Jy sample (Dicken et al. 2011). The spectrum shows prominent fine structure lines, as well as PAH and $\mathrm{H}_{2}$ emission features, and a silicate absorption band. The detection of emission lines in the mid-IR spectra of radio galaxies is common, and the presence of high ionisation potential lines, such as the [OIV] and [NeV] lines, clearly detected in PKS1814-63, indicates a significant AGN photoionised component (see e.g. Ogle et al. 2010, and references therein). However, the detection of $\mathrm{H}_{2}$ and $\mathrm{PAH}$ features is rare in radio galaxies in general - only 5 objects $(12 \%)$ in the 2 Jy sample show significant $\mathrm{H}_{2}$ emission, and only 10 objects (23\%) have detected PAH bands (Dicken et al. 2011).

The most prominent feature in the mid-IR spectrum of PKS 1814-637 is the $10 \mu \mathrm{m}$ silicate absorption feature. The absorption depth of the silicate feature in PKS 1814-637 is relatively high $\left(\tau_{10 \mu \mathrm{m}}=0.48\right)$ compared to the $40 \%$ of NLRG in the 2 Jy sample that show a silicate absorption feature in their midinfrared spectra. In the context of orientation based unification schemes for AGN, the detected silicate absorption is consistent with an AGN viewed edge-on that is obscured by circum-nuclear dust i.e. a NLRG. However, the $10 \mu \mathrm{m}$ silicate absorption feature in PKS 1814-637 is unique for CSS in the 2 Jy sample. This fact may be related to the large-scale dusty disk component that is more prominent in the case of PKS 1814-637 than in any other radio galaxy in the 2 Jy sample.

Overall, the mid-infrared spectral data for PKS 1814-637 provide evidence for the presence of a rich ISM, with a range of ionized gas, molecular and dust features detected. Although strong $\mathrm{PAH}$ and $\mathrm{H}_{2}$ features (but not silcate absorption: see above) appear to be a relatively common feature of CSS sources (Dicken et al. 2011), their detection is rare for powerful radio galaxies in general. Indeed, the overall character of the midIR spectrum of PKS 1814-637 - with its mix of high and low ionization fine structure, $\mathrm{H}_{2}$, silicate and PAH features - shows greater similarity to Seyfert galaxies (Gallimore et al. 2010; Baum et al. 2010) than it does to typical radio galaxies (Ogle et al. 2006; Dicken et al. 2011), as already pointed out in the previous section.

\section{The ISM of PKS 1814: radio continuum and $21-\mathrm{cm}$ atomic neutral hydrogen}

\subsection{Previous radio continuum VLBI observations}

PKS 1814-637 was observed in VLBI at $2.3 \mathrm{GHz}$ by Tzioumis et al. (2002). Figure 1 (right) shows the complex structure of the source which has an overall extent of $\sim 400$ mas (i.e. $\sim 480 \mathrm{pc}$ ). Two lobe-like structures with very different morphologies are observed: the southern region is dominated by two components with similar brightness embedded in a relatively large diffuse component, while the northern region shows a prominent bright component with possible North-South symmetrical extensions. Just over $50 \%$ of the total flux density of the source is detected in the VLBI observations, indicating the presence of a more diffuse extended component (but limited to the arcsec scale as no extended component was reported from the ATCA observations).

A weak radio continuum component is also observed (at the $5 \%$ level) between the two major lobes in the $2.3 \mathrm{GHz}$ image, which corresponds to a $15 \sigma$ detection. Interestingly, $8.4 \mathrm{GHz}$ VLBI observations (Ojha et al. 2004) confirm the presence and prominence of this component at high frequency, suggesting this 
Table 2. Summary of the kinematic data.

\begin{tabular}{|c|c|c|c|c|c|c|c|c|}
\hline (1) & (2) & $\begin{array}{l}\text { Velocity } \\
\text { width } \\
\left(\mathrm{km} \mathrm{s}^{-1}\right) \\
(3)\end{array}$ & $\begin{array}{c}\Delta \\
\left(\mathrm{km} \mathrm{s}^{-1}\right) \\
(4)\end{array}$ & $\begin{array}{c}\text { Velocity } \\
\text { shift } \\
\left(\mathrm{km} \mathrm{s}^{-1}\right) \\
(5)\end{array}$ & $\begin{array}{c}\Delta \\
\left(\mathrm{km} \mathrm{s}^{-1}\right) \\
(6)\end{array}$ & $\begin{array}{l}z \\
(7) \\
\end{array}$ & $\begin{array}{l}\Delta \\
(8) \\
\end{array}$ & (9) \\
\hline $\begin{array}{l}\text { Optical } \\
{[\mathrm{O} \text { III] }}\end{array}$ & $\begin{array}{c}\mathrm{n} \\
\mathrm{b} \\
1 \text { Gaussian }\end{array}$ & $\begin{array}{c}\text { unres } \\
569 \\
411\end{array}$ & $\begin{array}{l}35 \\
17\end{array}$ & $\begin{array}{c}+162 \\
0\end{array}$ & $\begin{array}{l}21 \\
20\end{array}$ & $\begin{array}{l}0.06466 \\
0.06412\end{array}$ & $\begin{array}{l}0.00007 \\
0.00014\end{array}$ & $\begin{array}{l}\text { H08 } \\
\text { H08 } \\
\text { H08 }\end{array}$ \\
\hline $\begin{array}{l}\text { Radio } \\
\text { H I }\end{array}$ & $\begin{array}{l}\mathrm{n} \\
\mathrm{b}\end{array}$ & $\begin{array}{c}\sim 50 \\
\sim 280^{a} \\
62 \dagger\end{array}$ & & $\begin{array}{c}-24 \\
162 \text { to }-119 \\
-192 \dagger\end{array}$ & & 0.06404 & & $\begin{array}{l}\text { M1 } \\
\text { M1 } \\
\text { V0† }\end{array}$ \\
\hline $\begin{array}{l}\text { Mid-IR } \\
{[\mathrm{Ne} \text { II }],[\mathrm{Ne} \text { III] }} \\
{\left[\mathrm{O} \text { IV], } \mathrm{H}_{2}\right.}\end{array}$ & 1 Gaussian & & & -87 & 60 & 0.06383 & 0.00020 & D11 \\
\hline $\begin{array}{l}\text { near-IR } \\
\text { Paschen } \alpha \\
{[\text { Fe II] }} \\
\text { S(3) } \\
\text { s(1) }\end{array}$ & & & & $\begin{array}{l}-126 \\
-6 \\
-36 \\
-36\end{array}$ & $\begin{array}{l}60 \\
30 \\
90 \\
60\end{array}$ & $\begin{array}{c}0.06370 \\
0.0641 \\
0.0640 \\
0.0640\end{array}$ & $\begin{array}{c}0.00015 \\
0.0001 \\
0.0003 \\
0.00015\end{array}$ & \\
\hline
\end{tabular}

Notes. Columns are: (1) measured emission/absorption line, (2) emission/absorption line component, $(3,4)$ velocity width (FWHM) and error in $\mathrm{km} \mathrm{s}^{-1},(5,6)$ velocity shift and error $\left(\mathrm{km} \mathrm{s}^{-1}\right)$ with respect to the assumed systemic velocity (taken to be the nuclear broad component of [O III]; see Sect. 3), (7, 8) redshift and error with respect to the broad component of [O III].

References. H08: Holt et al. (2008); M01: Morganti et al. (2001); D11: Dicken et al. (2011); V0: Véron-Cetty et al. (2000). ${ }^{(a)}$ Full width at zero intensity (FWZI) of the broad, shallow absorption. $\dagger$ Véron-Cetty et al. (2000) give e.g. FWZI and we have estimated the FWHM from their figures, but also give their data here.

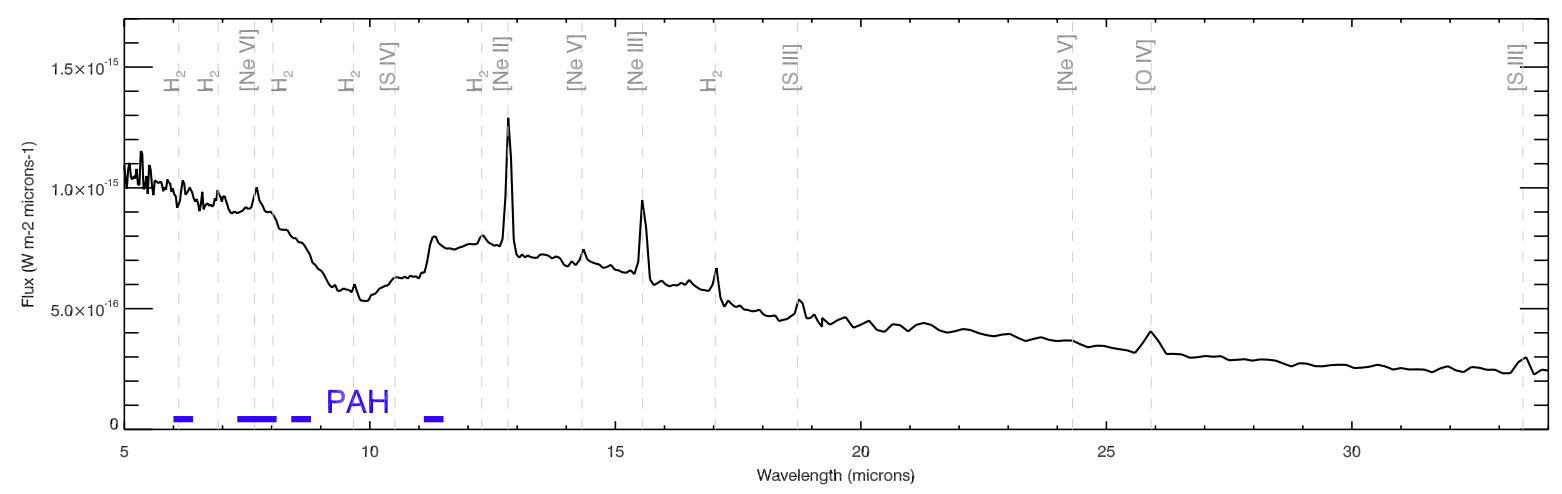

Fig. 6. Spitzer mid-infrared spectrum of PKS 1814-637. Fine structure lines are indicated as well as the position of strong PAH emission bands at $6.2,7.8,8.6$ and 11.3 microns. Note the strong 10 micron silicate absorption feature.

component is likely to be the radio core in PKS 1814-637 (see Fig. 1 right).

As a final remark, it is worth mentioning that the radio structure is not perpendicular to the galactic disk and dust lane (see Fig. 1) as in many dust lane galaxies (e.g. Cen A), but forms an angle of about $50^{\circ}$ (projected) to the galactic disk and dust lane.

\subsection{LBA observations: data reduction and radio continuum}

$\mathrm{HI}$ observations centred at $1336 \mathrm{MHz}$ were obtained with the Australian Long Baseline Array (LBA) on 27 Nov. 1998. The array comprised four stations; Parkes (64 m), Mopra (22 m), the Australia Telescope Compact Array $(5 \times 22-\mathrm{m}$ dishes as tiedarray), and the Mount Pleasant 26-m antenna of the University of Tasmania. We used a recording band of $8 \mathrm{MHz}$ width in each circular polarisation and 256 spectral channels. The editing and part of the calibration of the data was done in AIPS and then the data were transferred to MIRIAD (Sault et al. 1995) for the bandpass calibration. The calibration of the bandpass was done using additional observations of the strong calibrator PKS 1921-293. The resulting velocity resolution is $\sim 7 \mathrm{~km} \mathrm{~s}^{-1}$ before Hanning smoothing.

The line cube was made using uniform weighting after subtracting the continuum emission from the $u v$-data using the linefree channels. The noise per channel is $\sim 4 \mathrm{mJy}^{\text {beam }}{ }^{-1}$ after Hanning smoothing and the restoring beam size is $33 \times 16$ mas $\left(\mathrm{PA}=-72.4^{\circ}\right)$.

A continuum image was obtained using the line-free channels. The image is shown in Fig. 7 and it was obtained using AIPS and DIFMAP. The beam size is $36 \times 18$ mas $\left(\mathrm{PA}=-76^{\circ}\right)$. These 


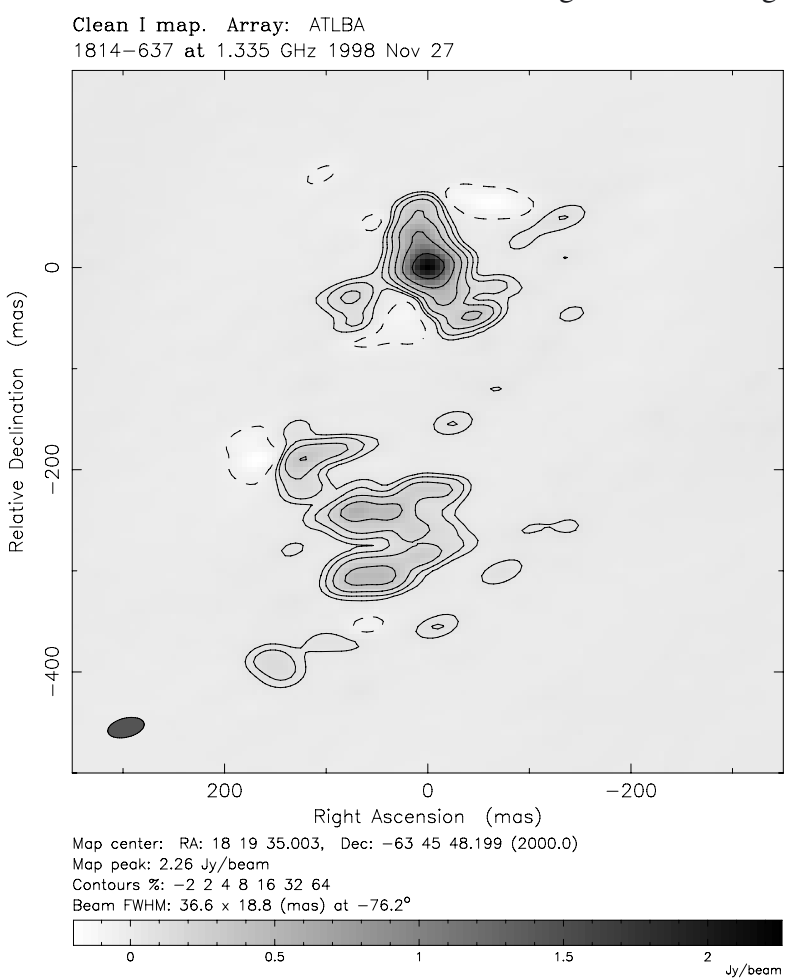

Fig. 7. PKS 1814-637 at $1335 \mathrm{MHz}$ as obtained from the line-free channels of the observations presented in this paper.

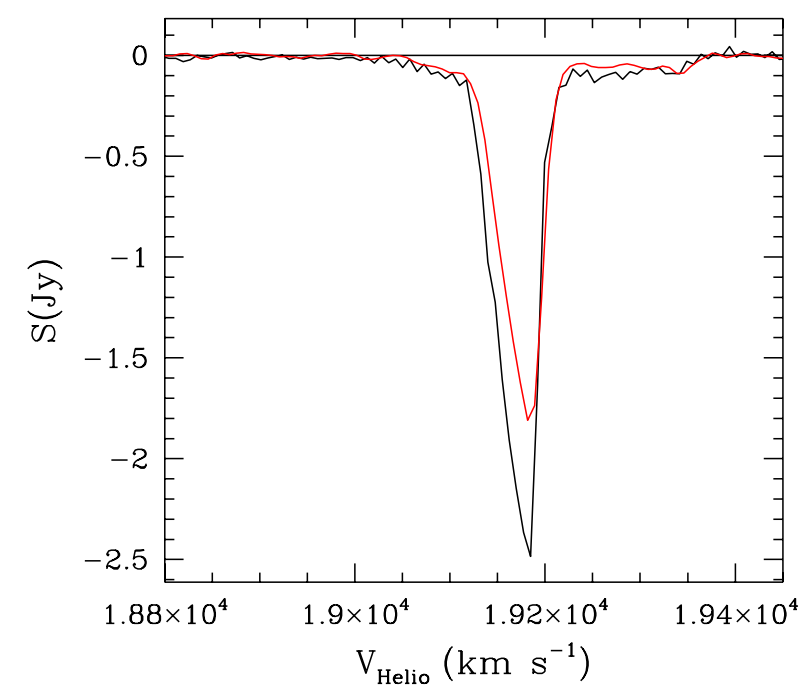

Fig. 8. Comparison of the ATCA (black) and LBA (red) integrated $\mathrm{HI}$ absorption profiles.

data were originally intended to provide information about the spectral index (in combination with the $2.3 \mathrm{GHz}$ data). However, the data quality prevented us from fully achieving this goal. The rms noise in the image is $\sim 12 \mathrm{mJy} /$ beam. Although the quality is clearly inferior to the $2.3 \mathrm{GHz}$ image of Tzioumis et al. (2002) - the rms noise is more than twice as high - the $1.3 \mathrm{GHz}$ image confirms the overall structure of the source.

The total flux detected is $S_{1.3 \mathrm{GHz}}=11.5 \mathrm{Jy}$. Compared with the $13.5 \mathrm{Jy}$ detected with ATCA observations it confirms that a fraction of the flux (at least 2 Jy, i.e. 15\%) is likely undetected because it originates in diffuse, low surface brightness emission. Because of the calibration problems mentioned above, we could only attempt an estimate of the integrated spectral index (using
$1.3 \mathrm{GHz}$ and $2.3 \mathrm{GHz}$ images convolved to the same restoring beam). We obtain values between $1.3 \mathrm{GHz}$ and $2.3 \mathrm{GHz}$ of $\alpha_{2.3}^{1.3} \sim-1.4$ for the southern lobe and $\alpha_{2.3}^{1.3} \sim-1.15$ for the northern lobe, consistent with our assumption that both structures are in fact radio lobes. Higher quality data will be necessary for an accurate estimate of the spectral index.

\subsection{Results from the HI absorption}

The LBA observations show that the H I absorption is extended and complex on the VLBI scale. The VLBI HI observations recover a large fraction of the absorbed flux observed at low resolution with the ATCA (see Fig. 8). The ATCA profile already suggested the presence of at least two components of H I absorption: a deep and relatively narrow component (with high optical depth, $\tau \sim 20 \%$ ) and broad and shallow wings. Interestingly, these components have, on the VLBI scale, different spatial distributions. As can be seen in Fig. 9, the deep absorption is extended and covers the entire source, while the shallow wings are more localised. The redshifted wing is observed only against the northern lobe while the blueshifted wing is detected against the southern lobe. The redshifted wing appears to be more prominent than the blueshifted one. The column density is $\sim 3 \times 10^{20} \mathrm{~cm}^{-2}$ for the deep component assuming a $T_{\text {spin }}$ (temperature that gives the relative population of the two levels) $\sim 100 \mathrm{~K}$.

As discussed in Sect. 3, the central velocity of the deep absorption is consistent with the results from the optical and IR emission lines and is defined as systemic velocity. Interestingly, the range in velocity covered by the $\mathrm{HI}$ in the broad wings is comparable to the velocity range seen in the ionised gas (see Fig. 4 and Table 2).

The H I absorption can be interpreted as coming from two separated gaseous structures, with the deep absorption due to cold gas located at large distance from the nucleus and radio source likely associated with the large-scale disk of the host galaxy. This would explain why this component is detected over the entire source and peaks around the systemic velocity. Furthermore, the high optical depth would be due to gas with a column density similar to that found in large scale disks, e.g. in Seyfert galaxies but also for radio-quiet early-type galaxies (Gallimore et al. 1999), and characterised by a $T_{\text {spin }} \sim 100 \mathrm{~K}$. Considering that the large-scale, galactic disk is seen edge-on, the fact that the deep component is projected over the entire VLBI source would imply a thickness of the disk of the order of 400-500 pc, comparable to what is expected for these types of structures in spiral galaxies (in particular in the outer regions where they tend to flare). The shallow, broad components could, instead, trace a circumnuclear disk located closer to the radio source: the broad widths of the shallow, shifted absorption features would be due to unresolved rotation or projected along the line of sight.

If this circumnuclear disk has approximately the same orientation as the large-scale one, the detection of a velocity gradient would then be due to the misalignment of the radio structure compared to the gaseous disk. This would explain the observed velocity gradient (redshifted against the northern lobe and blueshifted against the southern lobe). The low optical depth observed even on the VLBI scale for the broad component would not be due to effect of filling factor but could also be due to an higher spin temperature (i.e. $T_{\text {spin }} \gtrsim 1000 \mathrm{~K}$ ) of the gas in this structure due to the vicinity of the AGN. This would be consistent with what found for other compact sources (see e.g. Holt et al. 2006). 


\section{Discussion}

The analysis of multi-wavelength data (imaging and spectroscopy) has revealed that PKS 1814-637 is an intriguing radio galaxy. The modelling of the optical image has shown the presence of a prominent disk component, confirmed by the best fitting Sérsic $n=2$ profile. This is interesting given the high radio power of PKS 1814-637: this is the first radio galaxy of such power (FRII-like) found to reside in a disk dominated galaxy, and the only one of this type in a complete southern sample of powerful radio sources (2 Jy sample, see e.g. Ramos Almeida et al. 2011). This raises the question of how this disk galaxy succeeded in producing such a powerful radio source, and why this is not a more common phenomenon.

\subsection{PKS 1814-637: an "impostor" radio galaxy?}

Given the evidence for a rich ISM we have found for PKS 1814637 as well as its highly distorted radio morphology, we argue that the interaction of the radio plasma with the ISM must have had a major impact on the characteristics of this source.

In a study of a sample of nearby radio galaxies, Tadhunter et al. (2011) noted the high incidence of CSS/GPS sources in particular among the objects showing the presence of a young stellar population component in their host galaxies. This has been suggested to be the result of an observational selection effect where the strong interaction of the radio jets with the rich ISM, characteristic of objects resulting from major mergers, may influence the conversion of the jet power into radio luminosity. Due to the compression of the magnetic field and the increased density of particles, the radio luminosity will be boosted. This would make these objects more likely to enter a radio flux selected sample of objects. Indeed, the possibility of a variation of efficiency with which beam power is converted into radio emission has been also suggested by Gopal-Krishna \& Wiita (1991) in order to explain the dependence of linear size of powerful radio sources on redshift.

Thus, PKS 1814-637 could represent one of the best examples supporting this scenario and be an "imposter" in the $2 \mathrm{Jy}$ sample: an intrinsically lower power object that is selected in the sample because of the rich ISM that may contribute to the efficient conversion of jet power into radio emission.

This idea is also supported by the fact that, although PKS 1814-637 is classified as a NLRG on the basis of the optical spectrum (see Sect. 3), its [O III] luminosity is lower than one would expect on the basis of its radio power (Morganti et al. 1997; Holt et al. 2009). In Fig. 5 we plot [O III] emission line luminosity versus $5 \mathrm{GHz}$ radio luminosity for the $2 \mathrm{Jy}$ sample, where $L_{[\mathrm{OIII}]}$ is known to be a good indicator of AGN power. This shows that, on average, CSS/GPS sources lie below the the distribution of radio galaxies for any given $L_{\text {Radio }}{ }^{3}$, providing evidence that these objects have enhanced radio emission for their AGN power. It is notable that PKS 1814-637 has the lowest [O III] luminosity out of all the objects classified as NLRG including the other CSS/GPS sources - in the 2 Jy sample.

As discussed in Sect. 3, PKS 1814-637 has an emission line luminosity, but not equivalent width, similar to that of WLRG, as seen in Fig. 5. WLRG have also been shown to have intrinsically weak AGN for their radio power, and the properties of their local ISM are also hypothesised to boost the radio luminosity (Dicken et al. 2009).

\footnotetext{
${ }_{3}$ Similar results are found correlating $L_{\text {Radio }}$ with other AGN indicators in the mid-infrared i.e. [O IV] $25.89 \mu \mathrm{m}$ and $24 \mu \mathrm{m}$ luminosity.
}

In this context, it is interesting to note that large H I disks in a sample of radio galaxies have been found only around compact radio sources (Emonts et al. 2007). PKS 1814-637 could be another example in this trend, considering that the characteristics of the deep HI absorption suggests that it originates from a large scale disk (not seen in emission because the object is too far away and the sensitivity of the ATCA is too low). This suggests that there exists a group of compact radio sources with large reservoirs of cool gas, either originating from gas-rich major mergers or present in pre-existing disks, where the radio emission has been boosted, at least temporarily, by the interaction of the radio plasma with the rich ISM. Therefore, as the jets expand beyond the central regions of the galaxy that contains the rich ISM, their radio luminosities will decline until they drop below the flux limit of the particular radio sample being considered. Such a scenario leads to a short life time and agrees with the apparent rarity of objects like PKS 1814-637. In addition to this, because of the many characteristics that we have identified in common between PKS 1814-637 and Seyfert galaxies, this group of objects may even represent a "missing link" between radio galaxies and Seyferts.

\subsection{The lack of outflow}

If the conclusion about the systemic velocity is confirmed, it means that, unlike the majority of high radio power CSS/GPS (Holt et al. 2008), PKS 1814-637 does not show evidence for a fast outflow of ionised or atomic neutral gas. This may seem surprising if, as suggested in the previous section, the radio emission is really boosted by strong jet-cloud interactions.

The orientation of the source may help to explain the lack of evidence for an outflow: a relation between the orientation of the source and the amplitude of the outflow has been presented in Holt et al. (2009) (i.e. higher inclination lower outflow velocity). Being close to the plane of the sky, it could be more difficult to see any outflow produced along the radio axis in PKS 1814-63. However, if this is the case, we might expect to see strong line broadening effects at the site of the jet-cloud interaction (e.g. due to expansion of the radio lobes perpendicular to the radio jets). It is also possible that the emission line outflow (but not a neutral outflow) could be hidden by the near edge-on dusty disk at optical wavelengths. In this case the emission lines would represent more extended material that is illuminated by the AGN but not directly involved in the outflow.

One possible solution may be that the shock induced by the radio jets is so strong, and the gas heated to such high temperatures, that it does not have the chance to cool and to radiate in optical emission lines or absorb in HI. Therefore, we only see strong emission from the shock photoionized precursor gas. This is what we think may be happening in Coma A to the SE of the nucleus (Solórzano-Iñarrea \& Tadhunter 2003), where the jet appears to make a "direct hit" on a dense cloud (and the radio source is clearly deflected at this location), but despite the strong high ionization line emission associated with the cloud, we do not see any sign of kinematic disturbance in the emission line kinematics. If this is the case, one may expect to detect X-ray (bremsstrahlung) emission. New Chandra observations have been recently obtained and show a soft excess (Mingo, priv. comm.). The lack of adequate spatial resolution will likely make difficult to confirm whether this excess can be due to bremsstrahlung emission. 


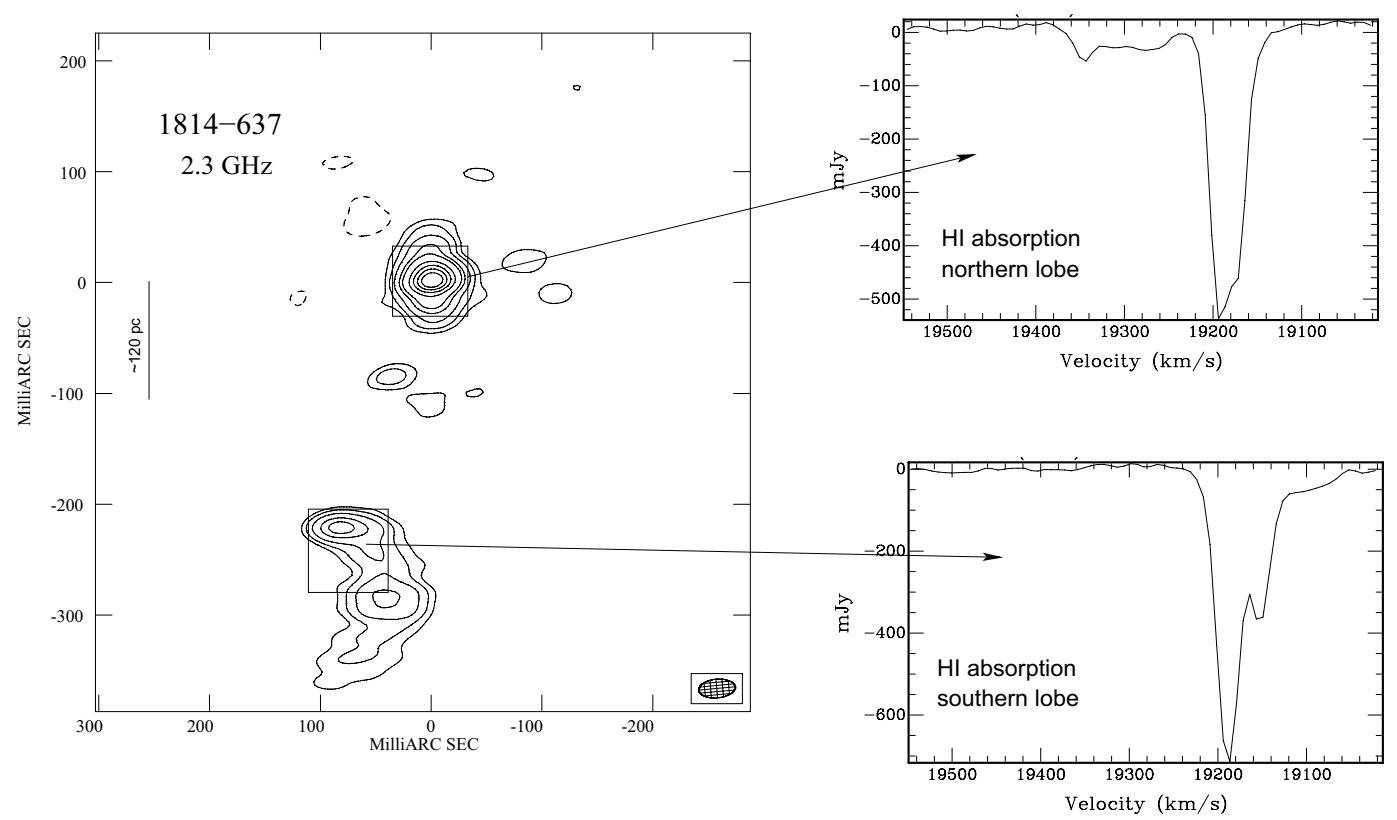

Fig. 9. VLBI 2.3 GHz image of PKS 1814-637 (left) from Tzioumis et al. (2002). The locations of the two integrated H I spectra shown on the right are marked. The two spectra clearly illustrate that the deep absorption is present at both locations while the broad, shallow absorption changes drastically going from the northern to the southern lobe.

\subsection{Link to Seyfert galaxies}

We have pointed out throughout this paper that similarities exist between PKS 1814-637 and Seyfert galaxies. We also remarked on the basis of our optical data in Sect. 3, as well as from our mid-IR data (Sect. 5), that without the information from the radio data, PKS 1814-637 would have been likely classified as a typical Seyfert galaxy in terms of its optical and mid-IR spectra, and optical morphology.

However, PKS 1814-637 has much higher radio power compared to even the most powerful Seyfert galaxies. In the case of radio-loud NLS1, that have been mentioned in the introduction, their radio power comparable to PKS 1814-637 (see e.g. Yuan et al. 2008; Foschini 2011, and references therein) is likely due to their radio emission is dominated by a beamed jet emission. Furthermore, in general the host galaxies of radio-loud NLS1 have not been yet well characterised and, therefore, it is not clear that these object are host by disk galaxies. Two examples (see Zhou et al. 2007; Foschini 2011) suggest that this could be the case at least for some of them. If this is confirmed for a larger sample, it may indicate a possible, interesting link between this group of object and objects like PKS 1814-637.

However, if we consider unbeamed, nearby Seyfert galaxies, even in those where clear evidence of jet-cloud interactions have been found, the radio emission does not reach the level observed in PKS 1814-637. An example is IC 5063, a radio-loud Seyfert galaxy where a strong ongoing interaction is observed between the radio plasma and the ISM (Oosterloo et al. 2000; Morganti et al. 2007, and references therein). The radio emission is aligned with the dust-lane, and the lobe interacting with a cloud of molecular gas is also much brighter in radio (Oosterloo et al. 2000). This object may represent another example of radio emission boosted by jet-cloud interaction, however the total radio power is almost two orders of magnitude lower than in PKS 1814-637.

Thus, it is unlikely that the difference between the radio power of Seyfert galaxies and that of PKS 1814-637 is solely due to the interaction; it is probable that the jet in PKS 1814-637 is intrinsically more powerful than in typical Seyfert galaxies, perhaps related to a higher bulge and black hole mass. To investigate this, we have attempted to estimate the mass of the black hole in PKS 1814-637 using different methods.

First, we have used the velocity gradient observed in the shallow H I component - that we have identified with a possible circumnuclear disk - as a probe of the $\mathrm{BH}$ mass. Ignoring projection effects that cannot be quantified, the $\mathrm{HI}$ velocities range from +180 to $-100 \mathrm{~km} \mathrm{~s}^{-1}$ relative to the systemic velocity. These velocities are measured at the location of the VLBI lobes, i.e. at a projected distance of about $100 \mathrm{pc}$ from the core. Under these conditions, we estimate a relatively high value for the $\mathrm{BH}$ mass in the range between $3 \times 10^{8}$ and $10^{9} \mathrm{M}_{\odot}$.

Alternatively, we can also make use of known $\mathrm{BH}-$ bulge mass relations Magorrian et al. (1998) and derive the BH mass from the bulge mass obtained from the $K$-band images (Inskip et al. 2010) and from the modelling of the galaxy. We have used the $K$-band magnitude from Inskip et al. (2010) to obtain a proxy and an upper limit to the bulge mass. The $K$-band magnitude $(K=12.52)$ is equivalent to an observed flux of $3.77 \times 10^{-16} \mathrm{erg} \mathrm{cm}^{-2} \mathrm{~s}^{-1} \mathrm{~A}^{-1}$. Assuming a galaxy with a $12 \mathrm{Gyr}$ old stellar population, the SED proposed by Maraston et al. (2009) and the luminosity distance of $\sim 265 \mathrm{Mpc}$, the observed flux matches a galaxy mass of $\sim 3 \times 10^{11} M_{\odot}$. Using this mass and the correlation shown in Fig. 2 of Häring \& Rix (2004), we derive a $\mathrm{BH}$ mass again in the range $\sim 3 \times 10^{8}$ to $\sim 10^{9} \mathrm{M}_{\odot}$, nicely consistent with that we derived from the HI. A similar value of $6 \times 10^{8} M_{\odot}$ is obtained by following instead Marconi \& Hunt (2003). These black hole mass estimates for PKS 1814637 are higher than those estimated for any of the nearby Seyfert galaxies in the sample of McLure \& Dunlop (2001, 2002), once corrected to our cosmology.

Finally, from the modelling of the optical images (see Sect. 2) we can derive an estimate of the bulge mass inside the derived effective radius $(6.45 \pm 0.01 \mathrm{kpc})$ using the integrated bulge magnitude $\left(r^{\prime}\right.$-band $\left.=15.75 \mathrm{mag}\right)$, and thus estimate a lower limit to the $\mathrm{BH}$ mass. We can use the absolute magnitude 
derived in this way $\left(M_{r}=-21.52\right)$ to extract, from the correlation shown in McLure \& Dunlop (2001), the BH mass. Again, the bulge luminosity of PKS 1814-637 appears to be located at the higher end of the distribution of Seyfert galaxies confirming that, unlike a typical Seyfert galaxy, PKS 1814-637 is hosted by a more early-type galaxy (S0-like), i.e. a galaxy with a large bulge. Following the prediction of Magorrian et al. (1998) (see also McLure \& Dunlop 2001 for details) the BH mass would be just below $\sim 10^{8} M_{\odot}$. However the plot in McLure \& Dunlop (2001) shows a large scatter in the value derived for Seyfert galaxies with comparable bulge mass, up to a few times $10^{8} \mathrm{M}_{\odot}$.

Thus, PKS 1814-637 appears to host a quite massive BH that would compare only with the most massive BH found in Seyfert galaxies. The combination of the strong interaction with the surround ISM discussed in the previous session and massive black hole (related to the large bulge of the host galaxy) could provide the right conditions to host a powerful radio AGN in this galaxy.

\section{Radio sources like PKS 1814-637: how rare?}

The case of PKS 1814-637 is particularly interesting because it is the first case of a powerful radio source in a disk galaxy. As described above, this allows us to understand the conditions in which a powerful radio source can be triggered even when hosted by a disk-like galaxy. In addition to this, it also helps our understanding of whether this type of AGN and radio source were more common in the earlier Universe. The idea of the interaction between radio plasma and dense ISM affecting the radio emission has indeed been proposed for high- $z$ radio galaxies to explain e.g. the correlation between the steepness of the spectral index and the redshift of the sources (Athreya \& Kapahi 1998; Klamer et al. 2006). The possibility of a higher incidence of powerful radio sources associated with disk galaxies at high- $z$ has been brought up by Norris et al. (2008), although they have so far identified only one possible candidate in their deep field. More recently, Schawinski et al. (2011) have studied the optical morphologies of host galaxies of AGN at $1.5<z<3$ X-ray selected $\left(10^{42}<L_{X}<10^{44} \mathrm{erg} \mathrm{s}^{-1}\right)$ in the CDF-S. The majority of these AGN are hosted by disk galaxies consistent with what is found for Seyfert galaxies in the local Universe (see also Cisternas et al. 2011).

Using the deep VLA radio observation presented by Mainieri et al. (2008), we find that two of the AGN selected by Schawinski et al. (2011) appear to have radio counterparts of 3.7 and $0.07 \mathrm{mJy}$ at $1.4 \mathrm{GHz}$. These sources are both at $z \sim 1.6$ and converting this to radio luminosity, these sources have a radio power of $\log P_{1.4 \mathrm{GHz}}=25.6 \mathrm{~W} / \mathrm{Hz}$ and $24.1 \mathrm{~W} / \mathrm{Hz}$ respectively. Considering the limited coverage of this deep field (at most $50 \mathrm{sq}$ armin) this represents an interesting result that may indicate that indeed high power radio sources hosted in disk galaxies could be more common at higher redshift.

The availability of large surveys coming on-line in the near future from new radio facilities (e.g. LOFAR, ASKAP, Apertif) should allow the existence of this population of sources to be confirmed.

Acknowledgements. C.R.A. acknowledges Christian Leipski for very useful comments on the GALFIT fitting. The Australia Telescope Compact Array (/Parkes telescope/Mopra telescope/Long Baseline array) is part of the Australia Telescope which is funded by the Commonwealth of Australia for operation as a National Facility managed by CSIRO. The authors would like to thanks the referee, Luigi Foschini, for his very constructive comments. This research has made use of the NASA Extragalactic Database (NED), whose contributions to this paper are gratefully acknowledged. This publication makes use of data products from the Two Micron All Sky Survey, which is a joint project of the University of Massachusetts and the Infrared Processing and Analysis Center/California Institute of Technology, funded by the National Aeronautics and Space Administration and the National Science Foundation.

\section{References}

Abdo, A. A., Ackermann, M., Ajello, M., et al. 2009, ApJ, 707, L142 Athreya R. M., \& Kapahi, V. K. 1998, JApA, 19, 63

Baum, S. A., Gallimore, J. F., O’Dea, C. P., et al. 2010, ApJ, 713, 708

Buttiglione, S., Capetti A., Celotti A., et al. 2010, A\&A, 509, A6

Best, P. N., Kauffmann, G., Heckman, T. M., et al. 2005, MNRAS, 362, 25

Bicknell, G. V., Dopita M. A., Tsvetanov Z. I., \& Sutherland R. S. 1998, ApJ, 495, 680

Cisternas, M., Jahnke, K., Inskip, K. J., et al. 2011, ApJ, 726, 57

de Vries 2009, Ph.D. Thesis, Leiden University

Dicken, D., Tadhunter, C., Axon, D., et al. 2009, ApJ, 694, 268

Dicken, et al. 2011, ApJ, submitted

Emonts, B. H. C., Morganti, R., Oosterloo, T. A., et al. 2007, A\&A, 464, L1

Emonts, B. H. C., Morganti, R., oosterloo, T. A., et al. 2008, MNRAS, 387, 197

Emonts, B. H. C., Tadhunter, C. N., Morganti, R., et al. 2009, MNRAS, 396 1522

Emonts B. H. C., Morganti, R., Struve, C., et al. 2010, MNRAS, 406, 987

Fanaroff, B. L., \& Riley, J. M. 1974, MNRAS, 167, 31

Fanti C. 2009, AN, 330, 120

Foschini, L. 2011, in Proc. Conf. Narrow-Line Seyfert 1 Galaxies and their place in the Universe published online at SISSA, Proc. Sci., 24

Gallimore, J. F., Baum, S. A., O’Dea C. P., Pedlar A., \& Brinks E. 1999, ApJ, 524,684

Gallimore, J. F., Yzaguirre, A., Jakoboski, J., et al. 2010, ApJS, 187, 172

Gopal-Krishna, \& Wiita, P. J. 1991, ApJ, 373, 325

Hardcastle, M. J., Evans, D. A., \& Croston, J. H. 2006, MNRAS, 370, 1893

Häring N., \& Rix, H.-W. 2004, ApJ, 604, L89

Heckman, T., Miley, G., van Breugel, W., \& Butcher, H. 1982, ApJ, 262, 529

Holt J., Tadhunter C., Morganti R., et al. 2006, MNRAS, 370, 1633

Holt, J., Tadhunter, C. N., \& Morganti, R. 2008, MNRAS, 387, 639

Holt, J., Tadhunter, C. N., \& Morganti, R. 2009, MNRAS, 400, 589

Hota, A., Sirothia, S. K., Ohyama, Y., et al. 2011, MNRAS, L304

Kanekar, N., \& Chengalur, J. N. 2008, MNRAS, 384, 6L

Keel, W. C., White, R. E., Owen, F. N., \& Ledlow, M. J. 2006, AJ, 132, 2233

Klamer, I. J., Ekers, R. D., Bryant, J. J., et al. 2006, MNRAS, 371, 852

Kunert-Bajraszewska, M., Marecki, A., \& Spencer, R. E. 2004, Proc. 7th Symp. of the European VLBI Network on New Developments in VLBI Science and Technologies, 73

Inskip, K. J., Tadhunter, C. N., Morganti, R., et al. 2010, MNRAS, 407, 1739

Lamer, G., Newsam, A. M., \& McHardy, I. M. 1999, MNRAS, 309, 1085

Ledlow, M. J., Owen, F. N., \& Keel, W. C. 1998, ApJ, 495, 227

Ledlow, M. J., Owen, F. N., Yun, M. S., \& Hill, J. M. 2001, ApJ, 552, 120

Magorrian, J., Tremaine, S., Richstone, D., et al. 1998, AJ, 115, 2285

Mainieri, V., Kellermann, K. I., Fomalont, E. B., et al. 2008, ApJS, 179, 95

Maraston, C., Strömbäck, \& G., Thomas D., Wake D. A., \& Nichol R. C. 2009 MNRAS, 394, L107

Marconi, A., \& Hunt, L. K. 2003, ApJ, 589, L21

McLure, R. J., \& Dunlop, J. S. 2001, MNRAS, 327, 199

McLure, R. J., \& Dunlop, J. S. 2002, MNRAS, 331, 795

Morganti, R., Killeen, N. E. B., \& Tadhunter, C. N. 1993, MNRAS, 263, 1023

Morganti, R., Tadhunter, C. N., Dickson, R., \& Shaw, M. 1997, A\&A, 326, 130

Morganti, R., Oosterloo, T. A., Tadhunter, C. N., et al. 2001, MNRAS, 323, 331

Morganti, R., Holt J., Saripalli, L., Oosterloo, T. A., \& Tadhunter, C. N. 2007, A\&A, 476, 735

Norris, R., Middelberg, E., \& Boyle, B. 2008, in From Planets to Dark Energy: the modern Radio Universe, October 1-5, 2007, The University of Manchester, UK, published online at SISSA, Proc. Sci., 50 [arXiv:0804.3998]

Ogle, P., Whysong, D., \& Antonucci, R. 2006, ApJ, 647, 161

Ogle, P., Boulanger F., Guillard P., et al. 2010, ApJ, 724, 1193

Oosterloo, T. A., Morganti, R., Tzioumis, A., et al. 2000, AJ, 119, 2085

Orienti, M., \& Dallacasa, D. 2010a, in Proc. 10th European VLBI Network Symposium an 2 EVN Users Meeting: VLBI and the new generation of radio array September 20-24, 2010, Manchester, UK, published online at SISSA, Proc. Sci., 40 [arXiv: 1012.0111]

Orienti, M., Murgia, M., \& Dallacasa, D. 2010b, MNRAS, 402, 1892

Ojha, R., Fey, A. L., Johnston, K. J., et al. 2004, AJ, 127, 3609

Peng, C. Y., Ho, L. C., Impey, C. D., \& Rix, H.-W. 2002, AJ, 124, 266

Peng, C. Y., Ho, L. C., Impey, C. D., \& Rix, H.-W. 2010, AJ, 139, 2097

Ramos Almeida, C., Tadhunter, C. N., Inskip, K. J., et al. 2011, MNRAS, 410, 1550 
R. Morganti et al.: The galactic environment of PKS 1814-637

Reynolds, C. S., \& Begelman, M. C. 1997, ApJ, 487, L135

Saikia, D. J., \& Jamrozy, M. 2009, BASI, 37, 63

Saikia, D. J., Jamrozy, M., Konar, C. \& Nandi, S. 2011, in Proc. 25th Texas Symposium on Relativistic Astrophysics, December 6-10, 2010, Heidelberg, Germany. ed. F. M. Rieger, C. van Eldik, \& W. Hofmann, published online at SISSA, Proc. Sci. [arXiv: 1104.1086]

Sault, R. J., Teuben, P. J., \& Wright, M. C. H. 1995, ASPC, 77, 433 Schawinski, K., Treister, E., Urry, C. M., et al. 2011, ApJ, 727, L31 Sérsic, J. L. 1963, BAAA, 6, 41

Solórzano-Iñarrea, C., \& Tadhunter, C. N. 2003, MNRAS, 340, 705

Stickel, M., \& Kühr, H. 1994, A\&AS, 105, 67

Sutherland, R. S., \& Bicknell, G. V. 2007, Ap\&SS, 311, 293

Tadhunter C. N., Morganti R., Robinson A., et al. 1998, MNRAS, 298, 1035
Tadhunter, C. N., Morganti, R., di Serego Alighieri, S., Fosbury, R. A. E., \& Danziger, I. J. 1993, MNRAS, 263, 999

Tadhunter, C., Holt, J., González Delgado, R., et al. 2011, MNRAS, 412, 960

Taylor, D., Dyson J. E., Axon, D. J., \& Pedlar, A. 1989, MNRAS, 240, 487

Tzioumis, A., King, E., Morganti, R., et al. 2002, A\&A, 392, 841

van Breugel, W., Heckman, T., Butcher, H., \& Miley, G. 1984, ApJ, 277, 82

Véron-Cetty, M.-P., \& Véron, P. 2001, A\&A, 375, 791

Véron-Cetty, M.-P., Woltjer, L., Stavely-Smith, L., \& Ekers, R. D. 2000, A\&A, 362,426

Zhou, H., Wang, T., Yuan, W., et al. 2007, ApJ, 658, L13

Wagner, A. Y., \& Bicknell, G. V. 2011, ApJ, 728, 29

Wright, E. L. 2006, PASP, 118, 1711

Yuan, W., Zhou H. Y., Komossa S., et al. 2008, ApJ, 685, 801 